\title{
Information and Conditional Probability to Go beyond Hidden Variables
}

\author{
Koji Nagata', Germano Resconi' ${ }^{2}$, Tadao Nakamura ${ }^{3}$, Han Geurdes ${ }^{4}$ \\ ${ }^{1}$ Department of Physics, Korea Advanced Institute of Science and Technology, Daejeon, South Korea \\ ${ }^{2}$ Department of Mathematics and Physics, Catholic University, Brescia, Italy \\ ${ }^{3}$ Department of Information and Computer Science, Keio University, Yokohama, Japan \\ ${ }^{4}$ Geurdes Data Science, Den Haag, The Netherlands \\ Email:ko_mi_na@yahoo.co.jp, resconi@speedyposta.it, nakamura@pipelining.jp, han.geurdes@gmail.com
}

How to cite this paper: Nagata, K., Resconi, G., Nakamura, T. and Geurdes, H. (2016) Information and Conditional Probability to Go beyond Hidden Variables. Journal of Applied Mathematics and Physics, 4, 22032214.

http://dx.doi.org/10.4236/jamp.2016.412214

Received: August 6, 2016

Accepted: December 17, 2016

Published: December 20, 2016

Copyright $\odot 2016$ by authors and Scientific Research Publishing Inc. This work is licensed under the Creative Commons Attribution International License (CC BY 4.0).

http://creativecommons.org/licenses/by/4.0/

\section{Open Access}

\begin{abstract}
We study the relation between the possibility of describing quantum correlation with hidden variables and the existence of the Bloch sphere. We derive some proposition concerning a quantum expected value under an assumption about the existence of the Bloch sphere in $N$ spin-1/2 systems. However, the hidden variables theory violates the proposition with a magnitude that grows exponentially with the number of particles. Therefore, we have to give up either the existence of the Bloch sphere or the hidden variables theory. We show that the introduction of curved information and the continuity equation of probability are in agreement with classical quantum mechanics. So we give up the hidden variable theory as local theory and we accept the Bloch sphere as global theory connected with the information space.
\end{abstract}

\section{Keywords}

Quantum Non Locality, Formalism

\section{Introduction}

The quantum theory (cf. [1]-[6]) gives approximate but frequently remarkably accurate numerical predictions. Much experimental data approximately have fit to the quantum predictions for the past some 100 years. We do not doubt the correctness of the quantum theory. The quantum theory also says new science with respect to information theory. The science is called the quantum information theory [6]. Therefore, the quantum theory gives us very useful another theory in order to create new information science and to explain the handling of raw experimental data in our physical world.

As for the foundations of the quantum theory, Leggett-type non-local variables 
theory [7] is experimentally investigated [8] [9] [10]. The experiments report that the quantum theory does not accept Leggett-type non-local variables interpretation. However, there are debates for the conclusions of the experiments. See Refs. [11] [12] [13].

As for the applications of the quantum theory, the implementation of a quantum algorithm to solve Deutsch's problem [14] on a nuclear magnetic resonance quantum computer is reported first [15]. The implementation of the Deutsch-Jozsa algorithm on an ion-trap quantum computer is also reported [16]. There are several attempts to use single-photon two-qubit states for quantum computing. Oliveira et al. implement Deutsch's algorithm with polarization and transverse spatial modes of the electromagnetic field as qubits [17]. Single-photon Bell states are prepared and measured [18]. In addition, the decoherence-free implementation of Deutsch's algorithm is reported using such single photon and using two logical qubits [19]. More recently, a one-waybased experimental implementation of Deutsch's algorithm is reported [20]. In 1993, the Bernstein-Vazirani algorithm was reported [21] [22]. It can be considered as an extended Deutsch-Jozsa algorithm. In 1994, Simon's algorithm was reported [23]. Implementation of a quantum algorithm to solve the Bernstein-Vazirani parity problem without entanglement on an ensemble quantum computer is reported [24]. Fiber-optics implementation of the Deutsch-Jozsa and Bernstein-Vazirani quantum algorithms with three qubits is discussed [25]. A quantum algorithm for approximating the influences of Boolean functions and its applications is recently reported [26]

We study the relation between a significant specific hidden variables theory and the existence of the Bloch sphere. The results of measurements are either +1 or -1 . We derive some proposition concerning a quantum expected value under an assumption about the existence of the Bloch sphere in $N$ spin-1/2 systems. However, the hidden variables theory violates the proposition with a magnitude that exponentially grows with the number of particles. Therefore, we have to give up either the existence of the Bloch sphere or the hidden variables theory. We solve the previous dilemma we introduce the information space and the continuity equation to show how quantum mechanics is consequence of the information locate in all the space so is impossible to represent by local hidden variables. In conclusion, we agree on the existence of the Bloch sphere.

\section{From Probability Continuity Equation and Information Space to Shrodinger Equation}

1) Continuity equation

Given the continuity equation of the probability (global conservation of the probability) we have

$$
\frac{\partial \rho}{\partial t}+\nabla \cdot(\rho v)=0
$$

when the probability density $\rho=R^{2}$ we have

$$
\frac{\partial R^{2}}{\partial t}+\nabla \cdot\left(R^{2} v\right)=2 R \frac{\partial R}{\partial t}+\nabla \cdot\left(R^{2} v\right)=2 R \frac{\partial R}{\partial t}+2 R \nabla R v+R^{2} \nabla v=0
$$


For $R \neq 0$ we have

$$
\frac{\partial R}{\partial t}+\nabla R v+\frac{1}{2} R \nabla v=\frac{\partial R}{\partial t}+\frac{1}{2 m}(2 \nabla R m v+R \nabla m v)
$$

Now in classical mechanics we have for the action $S$ the relation

$$
\nabla S=m v=p
$$

So

$$
\frac{\partial R}{\partial t}+\frac{1}{2 m}\left(R \nabla^{2} S+2 \nabla R \nabla S\right)=0
$$

where $S$ is the action and for the wave the phase of the wave.

2) Condition probability from statistical parameters (average value, standard deviation and others)

We assume that to found a particle in a particular state is a probabilistic phenomenon for which we have join probability that the particle can be in a particular state. Now the novelty is to assume that the probability is function of other external elements as parameters. The average of the position for the particle is a parameter the movement of the particle in a particular environment for example inside of a tube or in other boundary condition (see boundary condition in Shrodinger solution) can change the probability for a particular state. Any far or near change of the environment change the probability of the state (Bell theorem and entanglement) In conclusion the join probability of a state of different variables is conditioned by a set of parameters that statically or physically can define the environment where the particle move. We denote all this parameters as the information relate to the environment where the particle is located. The set of external parameters is the information space that can have curvature as in the Berry phase phenomena that show that in the Shrodinger solution any loop can change the original phase. In differential geometry any loop in a space with curvature changes the original phase of the vectors. Now we built the information space which geodesic tensor is the Fisher entropy or Fisher information by which we can compute the covariant derivatives and the curvature.

Given the system of the conditional probabilities

$$
\rho=\rho\left(x_{1}, \cdots, x_{q} \mid \theta_{1}, \cdots, \theta_{p}\right)
$$

We have the $p$ dimensional information reference

$$
e_{\alpha}=\left[\begin{array}{c}
\int_{\Omega} \frac{\partial \log \rho}{\partial \theta_{1}} \mathrm{~d} x \\
\int_{\Omega} \frac{\partial \log \rho}{\partial \theta_{2}} \mathrm{~d} x \\
\cdots \\
\int_{\Omega} \frac{\partial \log \rho}{\partial \theta_{p}} \mathrm{~d} x
\end{array}\right] .
$$

Given the vector 


$$
V=V^{\alpha} e_{\alpha}
$$

The derivative is

$$
\frac{\partial V}{\partial \theta^{\beta}}=\frac{\partial V^{\alpha}}{\partial \theta^{\beta}} e_{\alpha}+V^{\alpha} \frac{\partial e_{\alpha}}{\partial \theta^{\beta}}=\frac{\partial V^{\alpha}}{\partial \theta^{\beta}} e_{\alpha}+V^{\gamma} \frac{\partial e_{\gamma}}{\partial \theta^{\beta}}=\frac{\partial V^{\alpha}}{\partial \theta^{\beta}} e_{\alpha}+V^{\gamma} \int_{\Omega} \frac{\partial^{2} \log \rho}{\partial \theta^{\beta} \partial \theta^{\gamma}} \mathrm{d} x
$$

where

$$
\int_{\Omega} \frac{\partial^{2} \log \rho}{\partial \theta^{\beta} \partial \theta^{\gamma}} \mathrm{d} x=\Gamma_{\gamma \beta^{e} \alpha}^{\alpha}
$$

is the Fisher information matrix connected with the Christoffel symbols $\Gamma_{\gamma \beta}^{\alpha}$. For

$$
\frac{\partial^{2} \log \rho}{\partial \theta^{k} \partial \theta^{\gamma}} V^{\gamma}=\frac{\frac{\partial^{2} \log \rho}{\partial \theta^{k} \partial \theta^{\gamma}}}{\frac{\partial \log \rho}{\partial \theta^{k}} \frac{\partial \log \rho}{\partial \theta^{\gamma}}} \frac{\partial \log \rho}{\partial \theta^{k}}
$$

Here,

$$
V^{\gamma}=\frac{1}{\frac{\partial \log \rho}{\partial \theta^{\gamma}}}
$$

For the Fisher information we have

$$
\frac{E\left[\frac{\partial^{2} \log \rho_{j}}{\partial \theta^{p} \partial \theta^{\gamma}}\right]}{E\left[\frac{\partial \log \rho_{j}}{\partial \theta^{p}} \frac{\partial \log \rho}{\partial \theta^{\gamma}}\right]}=1
$$

where $E$ is the average operator so we can write in the first approximation

$$
D_{k}=\frac{\partial}{\partial \theta^{k}}-\frac{\partial \log \rho}{\partial \theta^{k}}
$$

and

$$
P_{k}=p_{k}-\frac{\partial \log \rho}{\partial \theta^{k}}
$$

where $p_{k}$ is the momentum. So we have

$$
\begin{aligned}
& \delta S=\delta \int \rho\left(\frac{\partial S}{\partial t}+\frac{1}{2 m} p_{i} p_{j}+V\right) \mathrm{d} t \mathrm{~d}^{n} x+\delta \frac{1}{2 m} \int \rho\left(\frac{\partial \log \rho}{\partial x_{i}} \frac{\partial \log \rho}{\partial x_{j}}\right) \mathrm{d}^{n} \mathrm{~d}^{n} x=0 \\
& \delta \int \rho\left(\frac{\partial S}{\partial t}+\frac{1}{2 m} p_{i} p_{j}+V\right) \mathrm{d} t \mathrm{~d}^{n} x \frac{\delta \rho\left(\frac{\partial S}{\partial t}+\frac{1}{2 m} p_{i} p_{j}+V\right)}{\partial \rho} \\
& -\frac{\partial}{\partial x_{\mu}} \frac{\delta \rho\left(\frac{\partial S}{\partial t}+\frac{1}{2 m} p_{i} p_{j}+V\right)}{\partial \frac{\partial \rho}{\partial x_{\eta}}}=\frac{\partial S}{\partial t}+\frac{1}{2 m} p_{i} p_{j}+V
\end{aligned}
$$

and 


$$
\begin{aligned}
& \delta \int \rho\left(\frac{\partial \log \rho}{\partial x_{i}} \frac{\partial \log \rho}{\partial x_{j}}\right) \mathrm{d} t \mathrm{~d}^{n} x=\frac{\partial\left(\rho \frac{\partial \log \rho}{\partial x_{i}} \frac{\partial \log \rho}{\partial x_{j}}\right)}{\partial \rho}-\frac{\partial}{\partial x_{\mu}} \frac{\partial\left(\rho \frac{\partial \log \rho}{\partial x_{i}} \frac{\partial \log \rho}{\partial x_{j}}\right)}{\partial \frac{\partial \rho}{\partial x_{\eta}}} \\
& =\frac{\partial \log \rho}{\partial x_{i}} \frac{\partial \log \rho}{\partial x_{j}}-\frac{1}{\rho} \frac{\partial\left(\frac{\partial \rho}{\partial x_{i}} \frac{\partial \rho}{\partial x_{j}}\right)}{\partial \frac{\partial \rho}{\partial x_{\mu}}}=\frac{1}{\rho^{2}} \frac{\partial \rho}{\partial x_{i}} \frac{\partial \rho}{\partial x_{j}}-\frac{1}{\rho} \frac{\partial}{\partial x_{\mu}} \frac{\partial\left(\frac{\partial \rho}{\partial x_{i}} \frac{\partial \rho}{\partial x_{j}}\right)}{\partial \frac{\partial \rho}{\partial x_{\eta}}} \\
& =\frac{1}{\rho^{2}} \frac{\partial \rho}{\partial x_{i}} \frac{\partial \rho}{\partial x_{j}}-\frac{2}{\rho} \frac{\partial^{2} \rho}{\partial x_{i} \partial x_{j}}=Q
\end{aligned}
$$

So

$$
\frac{\partial S}{\partial t}+\frac{1}{2 m} p_{i} p_{j}+V+\frac{1}{2 m}\left(\frac{1}{\rho^{2}} \frac{\partial \rho}{\partial x_{i}} \frac{\partial \rho}{\partial x_{j}}-\frac{2}{\rho} \frac{\partial^{2} \rho}{\partial x_{i} \partial x_{j}}\right)=\frac{\partial S}{\partial t}+\frac{1}{2 m} p_{i} p_{j}+V+Q=0
$$

Now for $R^{2}=\rho$ and the Plank constant is equal to 1 we have

$$
Q=-\frac{1}{2 m} \frac{\nabla^{2} R}{R}
$$

So we have

$$
\frac{\partial S}{\partial t}+\frac{|\nabla S|^{2}}{2 m}-\frac{1}{2 m} \frac{\nabla^{2} R}{R}+V=0
$$

where $Q$ is the Bohm quantum potential that is a consequence for the extreme condition of Fisher information (minimum or maximum condition for the Fisher information). We know that the quantum potential as real part and the continuous equation as the imaginary part from the Boltzmann entropic geometry we can generate the Schrödinger equation. We can also use the Schrodinger equation and came back to the Fisher information and to the pure conditional probability interpretation of the quantum mechanics.

Now, we combine the continuity equation of the probability with the covariant derivative in the curved information space we have

$$
\begin{aligned}
& \frac{\partial S}{\partial t}+\left(\frac{|\nabla S|^{2}}{2 m}-\frac{1}{2 m} \frac{\nabla^{2} R}{R}+V\right) \\
& \mathrm{i}\left(\frac{\partial R}{\partial t}\right)+\frac{1}{2 m}\left(R \nabla^{2} S+2 \nabla R \nabla S\right)=0
\end{aligned}
$$

where the real part is consequence of the curvature in the information space and the immaginary part is due to the continuous equation for the probability.

Now for $\Psi=\operatorname{Re}^{i S}$ the previous real and complex part are the real and immaginary of the classical Schrodinger equation.

$$
\mathrm{i} \frac{\partial \Psi}{\partial t}=\left(-\frac{1}{2 m} \nabla^{2}+V\right) \Psi
$$


with

$$
\frac{h}{2 \pi}=1
$$

In conclusion, we can make a reverse process used by Schrödinger we can generate the Schrödinger equation by the information space and the continuity equation of the probability. In this way, the Hilbert mechanism can be explain only by information, curvature and probability.

\section{A Hidden Variables Theory Does Not Meet the Existence of the Bloch Sphere}

Assume that we have a set of $N$ spins $\frac{1}{2}$. Each of them is a spin-1/2 pure state lying in the $x-y$ plane. Let us assume that one source of $N$ uncorrelated spin-carrying particles emits them in a state, which can be described as a multi spin-1/2 pure uncorrelated state. Let us parameterize the settings of the $j^{\text {th }}$ observer with a unit vector $\boldsymbol{n}_{j}$ (its direction along which the spin component is measured) with $j=1, \cdots, N$. One can introduce the 'hidden variables' correlation function, which is the average of the product of the hidden results of measurement

$$
E_{\mathrm{HV}}\left(\boldsymbol{n}_{1}, \boldsymbol{n}_{2}, \cdots, \boldsymbol{n}_{N}\right)=\left\langle r\left(\boldsymbol{n}_{1}, \boldsymbol{n}_{2}, \cdots, \boldsymbol{n}_{N}\right)\right\rangle_{\text {avg }},
$$

where $r$ is the hidden result. We assume the value of $r$ is \pm 1 (in $(\hbar / 2)^{N}$ unit), which is obtained if the measurement directions are set at $\boldsymbol{n}_{1}, \boldsymbol{n}_{2}, \cdots, \boldsymbol{n}_{N}$. We introduce ergodic averaging as a theoretical model here. We do not pursue the details of the assumption. To pursue the details is an interesting point. It is suitable to the next step of researches.

Also one can introduce a quantum correlation function with the system in such a pure uncorrelated state

$$
E_{\mathrm{QM}}\left(\boldsymbol{n}_{1}, \boldsymbol{n}_{2}, \cdots, \boldsymbol{n}_{N}\right)=\operatorname{tr}\left[\rho \boldsymbol{n}_{1} \cdot \boldsymbol{\sigma} \otimes \boldsymbol{n}_{2} \cdot \boldsymbol{\sigma} \otimes \cdots \otimes \boldsymbol{n}_{N} \cdot \boldsymbol{\sigma}\right]
$$

where $\otimes$ denotes the tensor product, $\cdot$ the scalar product in $\boldsymbol{R}^{2}, \sigma=\left(\sigma_{x}, \sigma_{y}\right)$ is a vector of two Pauli operators, and $\rho$ is the pure uncorrelated state,

$$
\rho=\rho_{1} \otimes \rho_{2} \otimes \cdots \otimes \rho_{N}
$$

with $\rho_{j}=\left|\Psi_{j}\right\rangle\left\langle\Psi_{j}\right|$ and $\left|\Psi_{j}\right\rangle$ is a spin-1/2 pure state lying in the $x$ - $y$ plane.

One can write the observable (unit) vector $\boldsymbol{n}_{j}$ in a plane coordinate system as follows:

$$
\boldsymbol{n}_{j}\left(\theta_{j}^{k_{j}}\right)=\cos \theta_{j}^{k j} \boldsymbol{x}_{j}^{(1)}+\sin \theta_{j}^{k j} \boldsymbol{x}_{j}^{(2)},
$$

where $\boldsymbol{x}_{j}^{(1)}=\boldsymbol{x}$ and $\boldsymbol{x}_{j}^{(2)}=\boldsymbol{y}$ are the Cartesian axes. Here, the angle $\theta_{j}^{k j}$ takes two values (two-setting model):

$$
\theta_{j}^{1}=0, \theta_{j}^{2}=\frac{\pi}{2}
$$


We derive a necessary condition to be satisfied by the quantum correlation function with the system in a pure uncorrelated state given in (27). In more detail, we derive the value of the product of the quantum correlation function, $E_{\mathrm{QM}}$ given in (27), i.e., $\left\|E_{\mathrm{QM}}\right\|^{2}$. We use the decomposition (29). We introduce simplified notations as

$$
T_{i_{1} i_{2} \cdots i_{N}}=\operatorname{tr}\left[\rho \boldsymbol{x}_{1}^{\left(i_{1}\right)} \cdot \boldsymbol{\sigma} \otimes \boldsymbol{x}_{2}^{\left(i_{2}\right)} \cdot \boldsymbol{\sigma} \otimes \cdots \otimes \boldsymbol{x}_{N}^{\left(i_{N}\right)} \cdot \boldsymbol{\sigma}\right]
$$

and

$$
\boldsymbol{c}_{j}=\left(c_{j}^{1}, c_{j}^{2}\right)=\left(\cos \theta_{j}^{k j}, \sin \theta_{j}^{k j}\right) .
$$

Then, we have

$$
\left\|E_{\mathrm{QM}}\right\|^{2}=\sum_{k_{1}=1}^{2} \cdots \sum_{k_{N}=1}^{2}\left(\sum_{i_{1}, \cdots, i_{N}=1}^{2} T_{i_{1} \cdots i_{N}} c_{1}^{i_{1}} \cdots c_{N}^{i_{N}}\right)^{2}=\sum_{i_{1}, \cdots, i_{N}=1}^{2} T_{i_{1} \cdots i_{N}}^{2} \leq 1,
$$

where we use the orthogonality relation $\sum_{k_{j}=1}^{2} c_{j}^{\alpha} c_{j}^{\beta}=\delta_{\alpha, \beta}$. The value of $\sum_{i_{1}, \cdots, i_{N}=1}^{2} T_{i_{1} \cdots i_{N}}^{2}$ is bounded as $\sum_{1 \cdots i_{N}=1}^{2} T_{i_{1} \cdots i_{N}}^{2} \leq 1$. We have

$$
\prod_{j=1 i_{j}=1}^{N}\left(\operatorname{tr}\left[\rho_{j} \boldsymbol{x}_{j}^{\left(i_{j}\right)} \cdot \boldsymbol{\sigma}\right]\right)^{2} \leq 1
$$

From the convex argument, all quantum separable states must satisfy the inequality (33). Therefore, it is a separability inequality. It is important that the separability inequality (33) is saturated iff $\rho$ is a multi spin-1/2 pure uncorrelated state such that, for every $j,\left|\Psi_{j}\right\rangle$ is a spin-1/2 pure state lying in the $x-y$ plane. The reason of the inequality (33) is due to the existence of the Bloch sphere in quantum mechanics

$$
\sum_{i_{j}=1}^{2}\left(\operatorname{tr}\left[\rho_{j} x_{j}^{\left(i_{j}\right)} \cdot \boldsymbol{\sigma}\right]\right)^{2} \leq 1
$$

The inequality (35) is saturated iff $\rho_{j}=\left|\Psi_{j}\right\rangle\left\langle\Psi_{j}\right|$ and $\left|\Psi_{j}\right\rangle$ is a spin-1/2 pure state lying in the $x-y$ plane. The inequality (33) is saturated iff the inequality (35) is saturated for every $j$. Thus, we have the maximal possible value of the scalar product as a quantum proposition concerning the Bloch sphere

$$
\left\|E_{\mathrm{QM}}\right\|^{2}=1
$$

when the system is in such a multi spin-1/2 pure uncorrelated state.

\subsection{Hidden Variables \& Reference Frames}

A hidden variables correlation function, assuming ergodicity, can be written as a weigthed sum over integer indices. For a function $r$ in a function space $\mathcal{R}$ we see e.g.

$$
E_{\mathrm{HV}}\left(\boldsymbol{n}_{1}, \cdots, \boldsymbol{n}_{N}\right)=\lim _{m \rightarrow \infty} \frac{1}{m} \sum_{\ell=1}^{m} r\left(\boldsymbol{n}_{1}, \cdots, \boldsymbol{n}_{N}, \ell\right)
$$

If a formulation with discrete indexing of hidden variables is a sensible way to describe a correlation, then one may select from $\mathcal{R}$ a function such that

$$
E_{\mathrm{HV}}(\overline{\bar{n}})=\lim _{m \rightarrow \infty} \frac{1}{m} \sum_{\lambda \in \mathcal{L}_{m}} r(\overline{\bar{n}}, \lambda)
$$


Here, the abbreviation $\overline{\bar{n}}=\left(\boldsymbol{n}_{1}, \cdots, \boldsymbol{n}_{N}\right)$ is used. Instead of an index, real variables are employed. In addition we may assume that the function space $\mathcal{R}$ contains a Heaviside type of function,

$$
f(x)=\lim _{K \rightarrow \infty} \exp \left[-\frac{\mathrm{e}^{-K x}}{K}\right] \in\{0,1\}
$$

A sign function can then be obtained $\propto f(x)-f(-x)$. Those sign functions are in $\mathcal{R}$ and, hence, $r$ can attain this form of sign function. The set $\mathcal{L}_{m}$ in (38) is a subset of the interval

$$
I=\left\{\lambda \in \mathbb{R} \mid-\infty<\ell_{I}<\lambda<u_{I}<\infty ; \ell_{I}, u_{I} \in \mathbb{R}\right\}
$$

and has cardinality $m$. More explicitly,

$$
\mathcal{L}_{m}=\left\{\lambda_{1}, \lambda_{2}, \cdots, \lambda_{m} ; \ell_{I}<\lambda_{1}<\lambda_{2}, \cdots<\lambda_{m}<u_{I}\right\}
$$

The elements of $\mathcal{L}_{m}$ can be shifted with an infinitesimal $\delta \lambda>0$. This gives, $\lambda^{\prime}=\lambda+\delta \lambda$, hence,

$$
\mathcal{L}_{m}^{\prime}=\left\{\lambda_{1}^{\prime}, \lambda_{2}^{\prime}, \cdots, \lambda_{m}^{\prime} ; \ell_{I}<\lambda_{1}^{\prime}<\cdots<\lambda_{m}^{\prime}<u_{I}\right\}
$$

and $\ell_{I}<\lambda_{1}<\lambda_{1}^{\prime}<\lambda_{2}<\lambda_{2}^{\prime} \cdots<u_{I}$.

\subsection{Maximum Value and Product}

We are very interested in the maximum value of the square of an expected value in a probability interpretation of quantum measurement theory. Therefore we focus on each measurement result providing a probability. And we study the maximum value when we inspect the summation. In short, we can multiply a measurement result by the same measurement result.

Therefore, we wish we would have some sort of Kronecker delta function at our disposal to match proper terms in the sum. In this respect it must be noted that the correlation in (38) can be arbitrary close approximated with the use of $\mathcal{L}_{m}^{\prime}$ in the sum. We have, still using the "=" symbol,

$$
E_{\mathrm{HV}}(\overline{\bar{n}})=\lim _{m \rightarrow \infty} \frac{1}{m} \sum_{\lambda^{\prime} \in \mathcal{L}_{m}^{\prime}} r\left(\overline{\bar{n}}, \lambda^{\prime}\right)
$$

Assuming, $m \rightarrow \infty$, squaring the $E_{\mathrm{HV}}$ is in close approximation equal to the product of expressions in (38) and (40)

$$
\left\{E_{\mathrm{HV}}(\overline{\bar{n}})\right\}^{2}=\frac{1}{m^{2}} \sum_{\lambda \in \mathcal{L}_{m}} \sum_{\lambda^{\prime} \in \mathcal{L}_{m}^{\prime}} r(\overline{\bar{n}}, \lambda) r\left(\overline{\bar{n}}, \lambda^{\prime}\right)
$$

Because, of small differences, we may write in a Taylor like approximation,

$$
r\left(\overline{\bar{n}}, \lambda^{\prime}\right)=r(\overline{\bar{n}}, \lambda)+\delta \lambda \frac{\partial r}{\partial \lambda}(\overline{\bar{n}}, \lambda)
$$

The $r$ product in (41) can then be re-written as

$$
r(\overline{\bar{n}} \lambda) r\left(\overline{\bar{n}}, \lambda^{\prime}\right)=r^{2}(\overline{\bar{n}}, \lambda)+\delta \lambda r(\overline{\bar{n}}, \lambda) \frac{\partial r}{\partial \lambda}(\overline{\bar{n}}, \lambda)
$$


If the previous result from (42) is introduced in (41) then the latter can be re-written as

$$
\left\{E_{\mathrm{HV}}(\overline{\bar{n}})\right\}^{2}=\frac{1}{m} \sum_{\lambda \in \mathcal{L}_{m}}\left[r^{2}(\overline{\bar{n}}, \lambda)+\delta \lambda r(\overline{\bar{n}}, \lambda) \frac{\partial r}{\partial \lambda}(\overline{\bar{n}}, \lambda)\right]
$$

If for $r \in \mathcal{R}$ a sign form is employed based on (39), then it is easy to see that for proper $\lambda \in \mathcal{L}_{m}$, the following

$$
0 \leq \frac{\partial r}{\partial \lambda}(\overline{\bar{n}}, \lambda) \leq 1
$$

will be true. The form $r(\overline{\bar{n}}, \lambda) \frac{\partial r}{\partial \lambda}(\overline{\bar{n}}, \lambda)$ contains, numerically and in limit, something similar to $x \delta(x)$ because $r$ is a sign $\propto f(x)-f(-x)$. We can conclude that given $r$ is a sign function based on (39), it is plausible to expect for proper $\mathcal{L}_{m}$ we obtain

$$
\left\{E_{\mathrm{HV}}(\overline{\bar{n}})\right\}^{2} \rightarrow \frac{1}{m} \sum_{\lambda \in \mathcal{L}_{m}} 1+O\left(\delta \lambda^{2}\right) \rightarrow 1+O\left(\delta \lambda^{2}\right)
$$

for $m \rightarrow \infty$.

\subsection{Quantum and $h v$ Values}

We study the possibility, $E_{\mathrm{QM}}=E_{\mathrm{HV}}$. For the ease of the presentation we write, $r(\lambda)$ and suppress the $\left(\boldsymbol{n}_{1}, \boldsymbol{n}_{2}, \cdots \boldsymbol{n}_{N}\right)$ notation. Hence, from our previous considerations we then write, under the limit, $m \rightarrow \infty$ and employing the result in (43)

$$
\left\|E_{Q M}\right\|^{2}=\sum_{k_{1}=1}^{2} \cdots \sum_{k_{N}=1}^{2}\left(\frac{1}{m} \sum_{\lambda \in \mathcal{L}_{m}} r(\lambda) \times \frac{1}{m} \sum_{\lambda^{\prime} \in \mathcal{L}_{m}^{\prime}} r\left(\lambda^{\prime}\right)\right) \rightarrow \sum_{k_{1}=1}^{2} \cdots \sum_{k_{N}=1}^{2}\left(1+O\left(\delta \lambda^{2}\right)\right) \rightarrow 2^{N} .
$$

We use the following fact

$$
\left(r\left(\boldsymbol{n}_{1}, \boldsymbol{n}_{2}, \cdots, \boldsymbol{n}_{N}, \lambda\right)\right)^{2}=1
$$

for properly selected $\mathcal{L}_{m}$. Hence, one has the following proposition concerning the hidden variables theory

$$
\left\|E_{Q M}\right\|^{2} \rightarrow 2^{N},(m \rightarrow \infty)
$$

Clearly, we cannot assign the truth value " 1 " for two propositions (36) (concerning the Bloch sphere) and (46) (concerning the hidden variables theory), simultaneously, when the system is in a multiparticle pure uncorrelated state. Of course we can imagine $\mathcal{L}_{m}$ where this would be possible but the $h_{v}$ theory would be in need of a sufficient number of special points in the $\mathcal{L}_{m}$ that makes the $r$ vanish for all $\overline{\bar{n}}$. In general, the claim can be made that the selection of $h v$ theories is not as free as one would prefer.

To continue we note, each of the theories refers to a spin-1/2 pure state lying in the $x$ - $y$ plane. Therefore, we are in the contradiction when the system is in such a multiparticle pure uncorrelated state. Thus, we cannot accept a general validity of the proposition of a hidden variables theory, if we assign the truth value " 1 " for the proposition (36) (concerning the Bloch sphere). 


\section{Conclusion and Discussions}

In conclusion, we have studied the relation between a hidden variables theory and the existence of the Bloch sphere and with a new type of conditional probability and Fisher information as metric for information space we show that the hidden variable are not possible. Now, we have derived some proposition concerning a quantum expected value under an assumption about the existence of the Bloch sphere in $N$ spin-1/2 systems. However, the hidden variables theory has violated the proposition with a magnitude that grows exponential with the number of particles. Therefore, we have had to give up either the existence of the Bloch sphere or the hidden variables theory. The hidden variables theory does not have depictured physical phenomena using the existence of the Bloch sphere with a violation factor that grows exponentially with the number of particles. Now, we point out the problem that when we cannot measure an observable we cannot say nothing on this measure as in the non-commutative case. So we have contradictions. In classical interpretation of quantum mechanics does not exist conditional probability and we cannot measure the probability but with the introduction of the information space and Fisher metric we show that conditional probability is possible but limited to statistical parameters as average value or other parameters. So contradiction is eliminated. Now, entanglement and Bell theorem can be understood in a new type of set theory that includes copula [27] and information [28]. Maybe we are right that projection operator is not sufficient to understand quantum mechanics so we cannot give Hilbert space axiomatic structure. Now, axiomatic Hilbert space is useful but cannot completely explain the meaning of the quantum mechanics. With information space, we can give a meaning with the axiomatic Hilbert that is always a useful mathematical instrument to use information and probability together.

\section{References}

[1] von Neumann, J. (1955) Mathematical Foundations of Quantum Mechanics. Princeton University Press, Princeton.

[2] Feynman, R.P., Leighton, R.B. and Sands, M. (1965) Lectures on Physics, Volume III, Quantum Mechanics. Addison-Wesley Publishing Company, Boston.

[3] Redhead, M. (1989) Incompleteness, Nonlocality, and Realism. 2nd Edition, Clarendon Press, Oxford.

[4] Peres, A. (1993) Quantum Theory: Concepts and Methods. Kluwer Academic, Dordrecht.

[5] Sakurai, J.J. (1995) Modern Quantum Mechanics. Revised Edition, Addison-Wesley Publishing Company, Boston.

[6] Nielsen, M.A. and Chuang, I.L. (2000) Quantum Computation and Quantum Information. Cambridge University Press, Cambridge.

[7] Leggett, A.J. (2003) Nonlocal Hidden-Variable Theories and Quantum Mechanics: An Incompatibility Theorem. Foundations of Physics, 33, 1469-1493.

https://doi.org/10.1023/A:1026096313729

[8] Gröblacher, S., Paterek, T., Kaltenbaek, R., Brukner, Č., Żukowski, M., Aspelmeyer, M. and Zeilinger, A. (2007) An Experimental Test of Non-Local Realism. Nature (London), 446, 871-875. https://doi.org/10.1038/nature05677 
[9] Paterek, T., Fedrizzi, A., Gröblacher, S., Jennewein, T., Żukowski, M., Aspelmeyer, M. and Zeilinger, A. (2007) Experimental Test of Nonlocal Realistic Theories without the Rotational Symmetry Assumption. Physical Review Letters, 99, Article ID: 210406.

https://doi.org/10.1103/PhysRevLett.99.210406

[10] Branciard, C., Ling, A., Gisin, N., Kurtsiefer, C., Lamas-Linares, A. and Scarani, V. (2007) Experimental Falsification of Leggett's Nonlocal Variable Model. Physical Review Letters, 99, Article ID: 210407. https://doi.org/10.1103/PhysRevLett.99.210407

[11] Suarez, A. (2008) Nonlocal "Realistic" Leggett Models Can Be Considered Refuted by the Before-Before Experiment. Foundations of Physics, 38, 583-589. https://doi.org/10.1007/s10701-008-9228-y

[12] Żukowski, M. (2008) Comment on: Nonlocal "Realistic" Leggett Models Can Be Considered Refuted by the Before-Before Experiment. Foundations of Physics, 38, 1070-1071. https://doi.org/10.1007/s10701-008-9250-0

[13] Suarez, A. (2009) On Bell, Suarez-Scarani, and Leggett Experiments: Reply to a Comment by Marek Żukowski in [Found. Phys. 38: 1070, 2008]. Foundations of Physics, 39, 156-159. https://doi.org/10.1007/s10701-008-9267-4

[14] Deutsch, D. (1985) Quantum Theory, the Church-Turing Principle and the Universal Quantum Computer. Proceedings of the Royal Society of London A, 400, 97-117. https://doi.org/10.1098/rspa.1985.0070

[15] Jones, J.A. and Mosca, M. (1998) Implementation of a Quantum Algorithm on a Nuclear Magnetic Resonance Quantum Computer. The Journal of Chemical Physics, 109, 16481653. https://doi.org/10.1063/1.476739

[16] Gulde, S., et al. (2003) Implementation of the Deutsch-Jozsa Algorithm on an Ion-Trap Quantum Computer. Nature, 421, 48-50. https://doi.org/10.1038/nature01336

[17] De Oliveira, A.N., Walborn, S.P. and Monken, C.H. (2005) Implementing the Deutsch Algorithm with Polarization and Transverse Spatial Modes. Journal of Optics B: Quantum and Semiclassical Optics, 7, 288-292. https://doi.org/10.1088/1464-4266/7/9/009

[18] Kim, Y.-H. (2003) Single-Photon Two-Qubit Entangled States: Preparation and Measurement. Physical Review A, 67, 040301(R).

[19] Mohseni, M., Lundeen, J.S., Resch, K.J. and Steinberg, A.M. (2003) Experimental Application of Decoherence-Free Subspaces in an Optical Quantum-Computing Algorithm. Physical Review Letters, 91, Article ID: 187903. https://doi.org/10.1103/PhysRevLett.91.187903

[20] Tame, M.S., Prevedel, R., Paternostro, M., Böhi, P., Kim, M.S. and Zeilinger, A. (2007) Experimental Realization of Deutsch's Algorithm in a One-Way Quantum Computer. Physical Review Letters, 98, Article ID: 140501. https://doi.org/10.1103/PhysRevLett.98.140501

[21] Bernstein, E. and Vazirani, U. (1993) Quantum Complexity Theory. Proceedings of the 25th Annual ACM Symposium on Theory of Computing (STOC '93), San Diego, 16-18 May 1993, 11-20. https://doi.org/10.1145/167088.167097

[22] Bernstein, E. and Vazirani, U. (1997) Quantum Complexity Theory. SIAM Journal on Computing, 26, 1411-1473.

[23] Simon, D.R. (1994) On the Power of Quantum Computation. Proceedings of 35th Annual Symposium on Foundations of Computer Science, Santa Fe, 20-22 November 1994, 116123.

[24] Du, J., et al. (2001) Implementation of a Quantum Algorithm to Solve the Bernstein-Vazirani Parity Problem without Entanglement on an Ensemble Quantum Computer. Physical Review A, 64, Article ID: 042306. https://doi.org/10.1103/PhysRevA.64.042306

[25] Brainis, E., Lamoureux, L.-P., Cerf, N.J., Emplit, P., Haelterman, M. and Massar, S. (2003) 
Fiber-Optics Implementation of the Deutsch-Jozsa and Bernstein-Vazirani Quantum Algorithms with Three Qubits. Physical Review Letters, 90, Article ID: 157902.

https://doi.org/10.1103/PhysRevLett.90.157902

[26] Li, H. and Yang, L. (2015) A Quantum Algorithm for Approximating the Influences of Boolean Functions and Its Applications. Quantum Information Processing, 14, 1787-1797. https://doi.org/10.1007/s11128-015-0954-8

[27] Resconi, G. and Nagata, K. (2016) Beyond Set Theory in Bell Inequality. Journal of Modern Physics, 7, 65-73. https://doi.org/10.4236/jmp.2016.71007

[28] Resconi, G., Licata, I. and Fiscaletti, D. (2013) Unification of Quantum and Gravity by Non Classical Information Entropy Space. Entropy, 15, 3602-3619.

\section{Submit or recommend next manuscript to SCIRP and we will provide best service} for you:

Accepting pre-submission inquiries through Email, Facebook, LinkedIn, Twitter, etc. A wide selection of journals (inclusive of 9 subjects, more than 200 journals)

Providing 24-hour high-quality service

User-friendly online submission system

Fair and swift peer-review system

Efficient typesetting and proofreading procedure

Display of the result of downloads and visits, as well as the number of cited articles

Maximum dissemination of your research work

Submit your manuscript at: http://papersubmission.scirp.org/

Or contact jamp@scirp.org 